\title{
The Use of Nuclear Imaging for Cardiac Resynchronization Therapy
}

\author{
Ji Chen • Mark M. Boogers • Jeroen J. Bax • \\ Prem Soman • Ernest V. Garcia
}

Published online: 23 February 2010

(C) The Author(s) 2010. This article is published with open access at Springerlink.com

\begin{abstract}
Cardiac resynchronization therapy (CRT) has shown benefits in patients with end-stage heart failure, depressed left ventricular (LV) ejection fraction $(\leq 35 \%)$, and prolonged QRS duration ( $\geq 120 \mathrm{~ms}$ ). However, based on the conventional criteria, $20 \%$ to $40 \%$ of patients fail to respond to CRT. Studies have focused on important parameters for predicting CRT response, such as LV dyssynchrony, scar burden, LV lead position, and site of latest activation. Phase analysis allows nuclear cardiology modalities, such as gated blood-pool imaging and gated myocardial perfusion single photon emission computed tomography (GMPS), to assess LV dyssynchrony. Most importantly, GMPS with phase analysis has the potential of assessing LV dyssynchrony, scar burden, and site of late activation from a single acquisition, so that this technique may provide a one-stop shop for predicting CRT response. This article provides a summary on the role of nuclear cardiology in selecting patients for CRT, with emphasis on GMPS with phase analysis.
\end{abstract}

Keywords Phase analysis · Gated SPECT - Myocardial perfusion imaging . Cardiac resynchronization therapy

\section{Introduction}

Heart failure (HF) is widely prevalent ( $>5$ million cases) and rapidly growing ( $>0.5$ million new cases annually) in the United States [1]. Hospital discharges rose from approximately 400,000 in 1979 to over 1 million in 2004 .

J. Chen $(\triangle) \cdot$ M. M. Boogers $\cdot$ J. J. Bax $\cdot$ P. Soman $\cdot$ E. V. Garcia Department of Radiology, Emory University School of Medicine, 1364 Clifton Road NE,

Atlanta, GA 30322, USA

e-mail: jchen22@emory.edu
Based on 44-year follow-up of the National Heart, Lung, and Blood Institute's Framingham Heart Study, $80 \%$ of men and $70 \%$ of women under 65 years of age who have HF will die within 8 years; in people diagnosed with HF, sudden cardiac death occurs at six to nine times the rate of the general population. The 2004 overall total death rate for HF was $52.0 \%$ [2]. In 2008, the estimated total cost of HF in the United States was $\$ 34.8$ billion [2].

Cardiac resynchronization therapy (CRT), provided by multisite pacing of the right and left ventricles, showed benefits in patients with end-stage HF. The benefits include improved HF symptoms, exercise capacity, quality-of-life score, and left ventricular (LV) function [3-8], as well as mortality benefits, in patients with advanced drug-refractory HF $[9,10]$. The American College of Cardiology/American Heart Association/Heart Rhythm Society guidelines recommend CRT in patients with end-stage drug-refractory HF of New York Heart Association (NYHA) class III or IV severity, depressed left ventricular ejection fraction (LVEF; $\leq 35 \%$ ), prolonged QRS duration ( $\geq 120 \mathrm{~ms}$ ), and sinus rhythm as a class I indication with level of evidence A [11]. However, using these conventional criteria for selecting patients for CRT, $20 \%$ to $40 \%$ of patients fail to respond to CRT [6, 7, 12-15]. It was suggested that electrical dyssynchrony represented by prolonged QRS intervals is not necessarily related to mechanical dyssynchrony, which may explain why $20 \%$ to $40 \%$ of the patients in the above trials did not respond to CRT [1618]. It is also possible that some patients who would have benefited from CRT were not included in the trials, such as patients with wide QRS complex who do not exhibit LV dyssynchrony and patients who have narrow QRS complex but who have LV dyssynchrony [17].

Echocardiography techniques, in particular two-dimensional echocardiography using color-coded tissue Doppler imaging 
(TDI), have been most widely used to measure LV dyssynchrony. These techniques have shown that LV mechanical dyssynchrony is an important predictor of response to CRT $[13,19,20]$. However, reliable TDI measurements require expertise to obtain reproducible results. Because of high intraobserver and interobserver variability, the PROSPECT (Predictors of Response to Cardiac Resynchronization Therapy) trial found that under "real-world" conditions the current available echocardiography techniques are not ready for routine practice to clinically predict CRT responses [21]. These results prompted the search for a more reproducible method of measuring LV dyssynchrony. Besides LV dyssynchrony, location and extent of viable or infarcted myocardium [22-24] and LV lead position [25, 26] were shown to be related to success of CRT.

Phase analysis allows nuclear cardiology modalities, such as gated blood-pool imaging and gated myocardial perfusion single photon emission computed tomography (SPECT; [GMPS]), to assess LV dyssynchrony. Phase analysis using GMPS (SyncTool, Emory University, Atlanta, GA) has evoked special interest because this technique has the potential for comprehensive assessment of multiple parameters (eg, LV dyssynchrony, myocardial scar burden and location, and site of latest activation) that influence response to CRT. This article provides a summary of the role of nuclear cardiology for selecting CRT candidates, with emphasis on GMPS with phase analysis.

\section{Gated Blood-Pool Imaging and Ventricular Dyssynchrony}

Phase analysis was first introduced with planar gated bloodpool ventriculography for evaluating the contraction pattern of the left ventricle [27-32]. Planar gated blood-pool images are acquired from one left anterior oblique view in different time frames, ranging from 16 to 64 frames per cardiac cycle. Regions of interest (ROI) are drawn on the planar images for left and right ventricles to generate time-activity curves, representing the variation of the ventricular volumes over the cardiac cycle. These time-activity curves are characterized by amplitude (height or depth of fitted curve) and phase angle (timing of contraction of a particular region). The standard deviation of the phase angles of the pixels in each ventricular ROI represents intraventricular dyssynchrony. The difference between the means of the phase angles of both ventricular ROI represents interventricular dyssynchrony. Toussaint et al. [30] evaluated the value of intraventricular and interventricular dyssynchrony measured from gated blood-pool ventriculography in 34 patients with end-stage HF undergoing CRT. All patients underwent gated blood-pool ventriculography at baseline and 6-month follow-up. Improvement of interventricular and intraventricular dyssynchrony was observed after CRT. Moreover, the combination of a baseline LVEF greater than $15 \%$ with significant interventricular dyssynchrony were the best predictors for improvement in LV systolic function after 6 months of CRT.

The major limitations of gated blood-pool ventriculography are overlap between adjacent structures and poor anatomic localization. In gated blood-pool SPECT, similar principles of phase analysis are used for detecting intraventricular and interventricular dyssynchrony. The gated blood-pool SPECT data are analyzed in three dimensions, resulting in better separation of adjacent structures and superior localization compared with gated blood-pool ventriculography [33-35]. A study including 19 patients with idiopathic dilated cardiomyopathy showed correlations between the clinical outcomes and the presence or absence of interventricular and intraventricular dyssynchrony, as measured by phase analysis of gated blood-pool SPECT [36]. This study also showed the improvement of interventricular and intraventricular dyssynchrony for patients with HF after CRT [36].

\section{Gated Myocardial Perfusion SPECT with Phase Analysis and LV Dyssynchrony}

\section{Technical Essentials}

Figure 1 shows the phase analysis of GMPS. The input to this tool is the standard gated SPECT short-axis image. At first, regional maximal count detection is performed in three dimensions for each temporal frame. Based on the partial volume effect [37], the variation of the regional maximal counts is proportional to the regional wall thickening over the cardiac cycle. The linear relationship was demonstrated in a phantom study [38]. Then, the firstharmonic Fourier function is used to approximate the discrete sample points into a continuous wall-thickening curve. For each region, the wall-thickening curve provides a phase angle that represents the onset of mechanical contraction of the region. Once the phase angles of all regions ( $>600$ regions over the entire left ventricle) are obtained, a phase distribution is generated that provides information on the degree of mechanical dyssynchrony for the entire left ventricle. The phase distribution can be displayed in polar map and in a histogram, as shown in Fig. 1. For a normal subject, the entire left ventricle starts contraction almost at the same time, so that the phase polar map is uniform and the phase histogram is narrow and highly peaked. Five quantitative indices are automatically calculated from the phase distribution: peak phase (the peak of the phase histogram), phase standard deviation (the standard deviation of the phase distribution), histogram bandwidth (the range of $95 \%$ of the phase angles), 


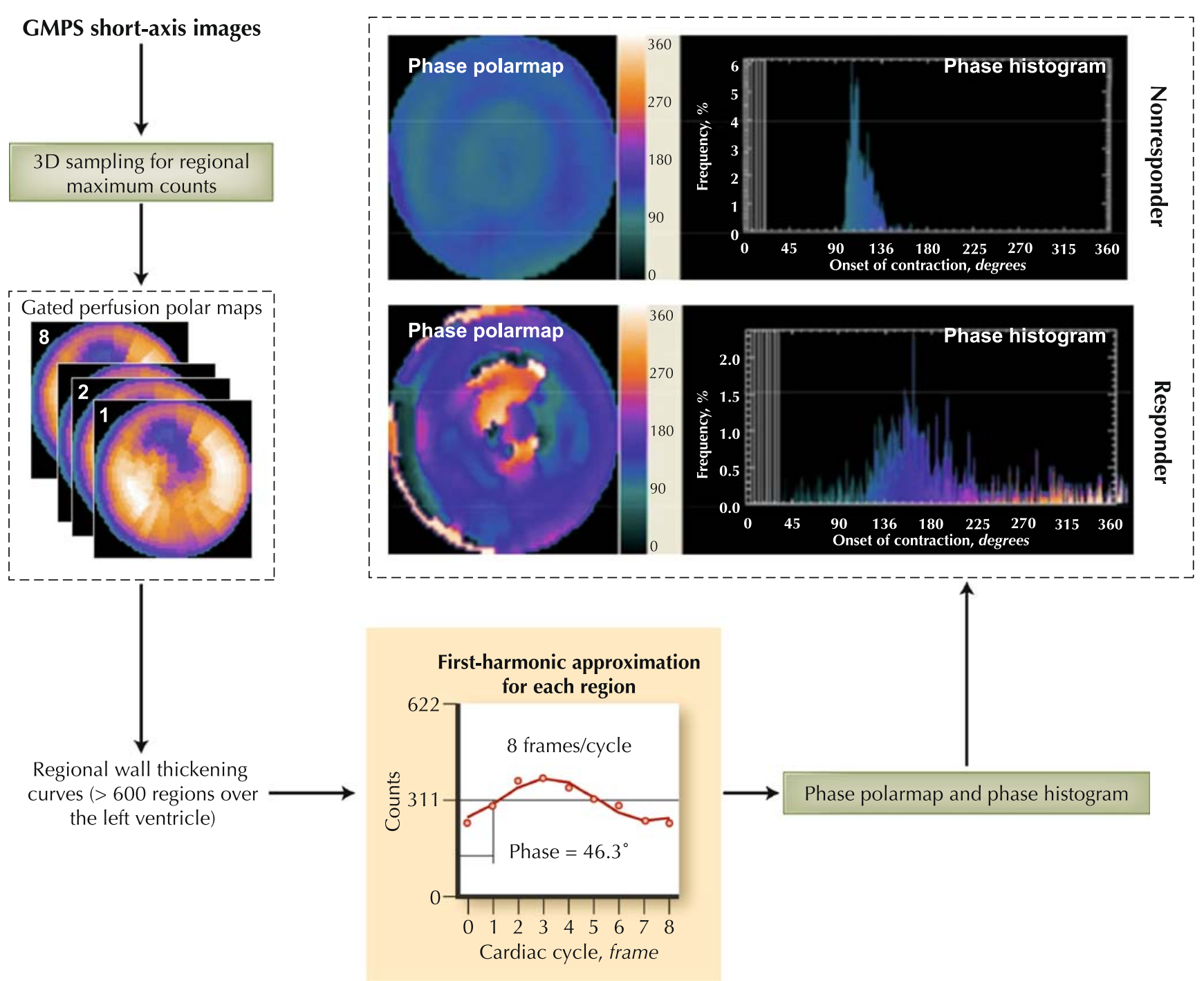

Fig. 1 Phase analysis of gated myocardial perfusion single photon emission computed tomography (GMPS) images. The inputs to phase analysis are the standard GMPS short-axis images. Three-dimensional (3D) sampling is performed on each temporal frame to detect the regional maximum counts. The variation of regional maximum counts over the cardiac cycle is proportional to wall thickening of the region. The points shown in the plots are the regional wall-thickening data. The first-harmonic Fourier function is used to approximate the wallthickening data (solid line) to calculate a phase angle for each region. Once the phase angles of all regions are obtained, a phase distribution

skewness (positive skewness means that the phase histogram is skewed to the right), and kurtosis (peakedness of the phase histogram) [39]. Among the five indices, histogram bandwidth and phase standard deviation (standard deviation of the phase distribution) are well studied for assessing LV dyssynchrony.

The phase analysis tool is largely automatic. Intraobserver and interobserver reproducibility of this technique have been evaluated in a study using 10 consecutive subjects with LV dysfunction ( $\mathrm{LVEF} \leq 35 \%)$ and 10 normal control subjects $[40 \bullet \cdot]$. For phase standard deviation and is generated and displayed in a polar map or in a histogram. Two examples are shown in this figure (a nonresponder and a responder to cardiac resynchronization therapy [CRT]). Both patients had New York Heart Association (NYHA) class III, depressed left ventricular (LV) ejection fraction $(<35 \%)$, and prolonged QRS duration $(>120 \mathrm{~ms})$. LV dyssynchrony was not present in the nonresponder, but present in the responder. Six months after CRT, the nonresponder deteriorated in NYHA class from III to IV, whereas the responder improved from class III to II

histogram bandwidth, intraobserver correlation coefficients were both 1.00 , and interobserver correlation coefficients were both 0.99 . The superior reproducibility of phase analysis is an advantage over echocardiography that may improve prediction of CRT response.

GMPS studies are often perceived to have a low temporal resolution, as the data are usually acquired using 8 to 16 frames per cardiac cycle. Because the discrete points of regional maximum counts are transformed into the continuous wall-thickening curves by the first-harmonic Fourier approximation, the actual temporal resolution of the 
phase analysis technique is greatly enhanced. A phantom simulation study demonstrated that with image quality achieved during routine clinical GMPS ( $\geq 10$ counts/pixel), the phase analysis tool was able to detect a minimum phase delay of $5.6^{\circ}$, representing $1 / 64$ of a cardiac cycle [41 ••].

\section{Clinical Validations}

GMPS with phase analysis was evaluated in a study comparing consecutive patients with LV dysfunction $(\mathrm{LVEF}<40 \% ; n=120)$, left bundle branch block $(n=33)$, right bundle branch block $(n=19)$, ventricular paced rhythms $(n=23)$, and normal control subjects $(n=157)$ to demonstrate its feasibility to detect LV dyssynchrony [42]. Phase standard deviation and histogram bandwidths were significantly different between the patient cohorts and the normal control subjects. Another study including 125 consecutive patients with LVEF less than 35\% showed that patients with prolonged QRS duration, on average, had significantly more LV dyssynchrony than patients without prolonged QRS duration [43]. However, the correlation between QRS duration and phase analysis results (phase standard deviation and histogram bandwidth) was weak. This finding confirmed that electrical dyssynchrony is not necessarily related to mechanical dyssynchrony, as was also suggested by an echocardiography study [44].

Henneman et al. [45.•] reported on the agreement between GMPS and two-dimensional echocardiography using TDI for the detection of LV dyssynchrony in 75 patients undergoing CRT. Good correlations were found between LV dyssynchrony measured with TDI and histogram bandwidth $(r=0.89, P<0.0001)$ and phase standard deviation $(r=0.80, P<0.0001)$ measured with GMPS. GMPS with phase analysis has also shown good correlation with three-dimensional (3D) echocardiography for assessing LV dyssynchrony [46, 47]. In 40 consecutive patients with HF, good agreement was found between standard deviation of time-to-peak systolic velocity (Ts-SD) on 3D echocardiography and histogram bandwidth $(r=0.77, P<$ $0.0001)$ and phase standard deviation $(r=0.74, P<0.0001)$ measured with phase analysis of GMPS. Patients with substantial LV dyssynchrony (Ts-SD $\geq 33 \mathrm{~ms}$ ) on 3D echocardiography showed significantly higher histogram bandwidth $\left(186^{\circ} \pm 52^{\circ}\right.$ vs $\left.74^{\circ} \pm 24^{\circ} ; P<0.0001\right)$ and phase standard deviation $\left(55.3^{\circ} \pm 13.6^{\circ}\right.$ vs $\left.25.1^{\circ} \pm 7.6^{\circ} ; P<0.0001\right)$ compared to patients without substantial LV dyssynchrony (Ts-SD $<33 \mathrm{~ms}$ ).

Henneman et al. [48••] evaluated whether GMPS with phase analysis can predict clinical response after 6 months of CRT in 42 patients with end-stage HF. Based on the improvement of $\geq$ one NYHA functional class, 30 patients were classified as responders and the other 12 patients as nonresponders. Both histogram bandwidth $\left(175^{\circ} \pm 63^{\circ}\right.$ vs $\left.117^{\circ} \pm 51^{\circ} ; P<0.01\right)$ and phase standard deviation $\left(56.3^{\circ} \pm\right.$ $19.9^{\circ}$ vs $\left.37.1^{\circ} \pm 14.4^{\circ} ; P<0.01\right)$ were significant higher in responders compared with nonresponders. Moreover, the optimal cutoff values of histogram bandwidth $\left(135^{\circ}\right)$ and phase standard deviation $\left(43^{\circ}\right)$ for predicting CRT response were derived by receiver operating characteristic curve analysis. With these optimal cutoff values, GMPS with phase analysis showed sensitivity/specificity values of $70 \%$ and $74 \%$, respectively, in predicting clinical response to CRT. Figure 1 shows an example of phase analysis in a nonresponder and a responder to CRT. Both patients had NYHA functional class III, depressed LVEF $(<35 \%)$, and prolonged QRS duration (>120 ms). LV dyssynchrony as measured by GMPS with phase analysis was not present in the nonresponder, but present in the responder. Six months after CRT, the nonresponder deteriorated in NYHA functional class from III to IV, whereas the responder improved from class III to II.

Phase analysis was recently implemented in the Quantitative Gated SPECT (Cedars Sinai Medical Center, Los Angeles, CA) software. Preliminary results for predicting CRT responses are similar to those detailed above $[49,50]$.

\section{Predictors of CRT Response in Addition to LV Dyssynchrony}

\section{Myocardial Scar Burden and Location}

The presence, location, and extent of myocardial scar from prior myocardial infarction have been shown to influence CRT response. Adelstein and Saba [24] studied the relationship between scar burden and CRT response. Fifty patients with end-stage drug-resistant HF and angiographically proven coronary artery disease were enrolled. At baseline, all patients had echocardiography and myocardial perfusion SPECT. Six months after CRT, all patients had echocardiography to evaluate cardiac reverse remodeling. CRT response was defined as a decrease of $\geq 15 \%$ in LV end-systolic volume from the pre-CRT to post-CRT echocardiography measurements. Among the 50 patients, 28 showed response to CRT and had lower perfusion defect score $(18.8 \pm 11.3$ vs $33.7 \pm 11.1 ; P<0.01)$ and scar density near the LV lead $(0.70 \pm 0.91$ vs $1.64 \pm 0.82 ; P<0.01)$ than the other 22 nonresponders. An inverse relationship was observed between global scar burden and absolute $(r=-0.63$, $P<0.01)$ or relative $(r=-0.53, P<0.01)$ increase in LVEF after 6 months post CRT. Moreover, the presence of myocardial scar tissue adjacent to the LV lead position was negatively correlated with increase in LVEF at 6 months post CRT. Similar results were reported by Ypenburg et al. [23] in 52 patients with ischemic HF and substantial LV dyssynchrony undergoing CRT. 
Bleeker et al. [22] reported the relationship between the location and transmurality of myocardial scar and CRT response in 40 patients with moderate-to-severe HF, $\mathrm{LVEF} \leq 35 \%$, prolonged QRS duration $(>120 \mathrm{~ms}$ ), and coronary artery disease. Patients with transmural infarction adjacent to the LV lead position (mostly the posterolateral region) showed a significantly lower response rate to CRT compared to patients without transmural scar tissue adjacent to the LV lead position.

\section{Site of Latest Activation}

The benefits of CRT are primarily based on synchronization of the myocardial contraction pattern, resulting in an improvement of LV systolic function. One important issue that may influence CRT response is the relationship between LV lead position and the site of latest activation. The site of latest activation in the left ventricle can vary substantially, and patients with an LV lead positioned away from the site of latest activation had suboptimal or no response to CRT [25]. Ypenburg et al. [26] evaluated echocardiographic and clinical outcome after 6 months post CRT in a large cohort of patients with ischemic or dilated cardiomyopathy. A total of $153(60 \%)$ patients had LV lead positioned at or adjacent to the site of latest activation based on chest radiographs. These patients showed a significant decrease in LV end-systolic and end-diastolic volumes along with an increase in LVEF after 6 months post CRT. The other $40 \%$ of the patients did not have concordant LV lead position and site of latest activation, and demonstrated no evident improvement in LV end-systolic and end-diastolic volumes and LVEF post CRT. This study also showed that mortality rate was significant lower in patients with concordant LV lead position and site of latest activation compared to patients without concordant LV lead position and site of latest activation at 24 months of followup $(15 \%$ vs $21 \%, P=0.048)$.

\section{GMPS with Phase Analysis: A Potential One-Stop Shop}

Phase analysis is a mathematical algorithm that applies to conventional GMPS data and does not need additional acquisition. This characteristic allows integrated analysis of multiple predictors of CRT response from a single GMPS study. Currently, phase standard deviation and histogram bandwidth are validated as the quantitative indices of global LV dyssynchrony. Because GMPS with phase analysis produces a phase distribution containing more than 600 regions over the left ventricle, it is possible to measure regional contraction delays and detect the site of latest activation. Then, the viability of the detected site of latest activation can be analyzed using the same perfusion image, so that an optimal LV lead position for the patient may be derived. Therefore, conceptually, GMPS with phase analysis has the potential of providing a one-stop shop for predicting CRT response by assessing LV dyssynchrony, myocardial scar burden and location, and site of latest activation.

\section{Conclusions}

Nuclear cardiology modalities, such as gated blood-pool imaging and GMPS, are promising alternatives for measuring LV dyssynchrony and predicting CRT response. Most importantly, GMPS with phase analysis has the potential for assessing LV dyssynchrony, myocardial scar burden and location, and site of latest activation from a single GMPS acquisition. Integrated analysis of these parameters, once validated, can be a viable clinical approach to consistently and reproducibly predict CRT response in patients with HF.

Disclosure Drs. Ji Chen and Ernest Garcia receive royalties from the sale of the Emory Cardiac Toolbox. The terms of this arrangement have been reviewed and approved by Emory University in accordance with its conflict-of-interest practice. The research in phase analysis is supported in part by a National Institutes of Health/National Heart, Lung, and Blood Institute-funded research project (1R01HL09443801A1, PI: Ji Chen). Dr. Mark Boogers is supported by the Dutch Heart Foundation grant number 2006 T102. Dr. Jeroen Bax receives research grants from Medtronic, Boston Scientific, Biotronik, Edwards Lifesciences, BMS medical imaging, St. Jude Medical, and GE Healthcare. Dr. Prem Soman receives a research grant from the American Society of Nuclear Cardiology Foundation for research in measuring acute CRT response by phase analysis.

Open Access This article is distributed under the terms of the Creative Commons Attribution Noncommercial License which permits any noncommercial use, distribution, and reproduction in any medium, provided the original author(s) and source are credited.

\section{References}

Papers of particular interest, published recently, have been highlighted as follows:

•- Of Major Importance

1. Rosamond W, Flegal K, Furie K, et al.: Heart disease and stroke statistics-2008 update: a report from the American Heart Association Statistics Committee and Stroke Statistics Subcommittee. Circulation 2008, 117:e25-e146.

2. American Heart Association: Heart Disease and Stroke Statistics: 2008 Update. Dallas, TX: American Heart Association; 2008.

3. Cazeau S, Leclercq C, Lavergne T, et al.: Effects of multisite biventricular pacing in patients with heart failure and intraventricular conduction delay. N Engl J Med 2001, 344:873-880.

4. Yu CM, Chau E, Sanderson JE, et al.: Tissue Doppler echocardiographic evidence of reverse remodeling and improved synchronicity by simultaneously delaying regional contraction after biventricular pacing therapy in heart failure. Circulation 2002, 105:438-445. 
5. Auricchio A, Stellbrink C, Sack S, et al.: Long-term clinical effect of hemodynamically optimized cardiac resynchronization therapy in patients with heart failure and ventricular conduction delay. $\mathrm{J}$ Am Coll Cardiol 2002, 39:2026-2033.

6. Abraham WT, Fisher WG, Smith AL, et al.: Cardiac resynchronization in chronic heart failure. N Engl J Med 2002, 346:1845-1853.

7. Young JB, Abraham WT, Smith AL, et al.: Combined cardiac resynchronization and implantable cardioversion defibrillation in advanced chronic heart failure: the MIRACLE ICD Trial. JAMA 2003, 289:2685-2694.

8. Auricchio A, Stellbrink C, Butter C, et al.: Clinical efficacy of cardiac resynchronization therapy using left ventricular pacing in heart failure patients stratified by severity of ventricular conduction delay. J Am Coll Cardiol 2003, 42:2109-2116.

9. Bristow MR, Saxon LA, Boehmer J, et al.: Cardiacresynchronization therapy with or without an implantable defibrillator in advanced chronic heart failure. N Engl J Med 2004, 350:2140-2150.

10. Cleland JG, Daubert JC, Erdmann E, et al.: The effect of cardiac resynchronization on morbidity and mortality in heart failure. $\mathrm{N}$ Engl J Med 2005, 352:1539-1549.

11. Epstein AE, DiMarco JP, Ellenbogen KA, et al.: ACC/AHA/HRS 2008 Guidelines for Device-Based Therapy of Cardiac Rhythm Abnormalities: a report of the American College of Cardiology/ American Heart Association Task Force on Practice Guidelines (Writing Committee to Revise the ACC/AHA/NASPE 2002 Guideline Update for Implantation of Cardiac Pacemakers and Antiarrhythmia Devices): developed in collaboration with the American Association for Thoracic Surgery and Society of Thoracic Surgeons. Circulation 2008, 117:e350-e408.

12. Bax JJ, Van der Wall EE, Schalij MJ: Cardiac resynchronization therapy for heart failure. N Engl J Med 2002, 347:1803-1804.

13. Bax JJ, Bleeker GB, Marwick TH, et al.: Left ventricular dyssynchrony predicts response and prognosis after cardiac resynchronization therapy. J Am Coll Cardiol 2004, 44:1834-1840.

14. Yu CM, Fung JW, Zhang Q, et al.: Tissue Doppler imaging is superior to strain rate imaging and postsystolic shortening on the prediction of reverse remodeling in both ischemic and nonischemic heart failure after cardiac resynchronization therapy. Circulation 2004, 110:66-73.

15. Yu CM, Zhang Q, Chan YS, et al.: Tissue Doppler velocity is superior to displacement and strain mapping in predicting left ventricular reverse remodelling response after cardiac resynchronisation therapy. Heart 2006, 92:1452-1456.

16. Leclercq C, Faris O, Tunin R, et al.: Systolic improvement and mechanical resynchronization does not require electrical synchrony in the dilated failing heart with left bundle-branch block. Circulation 2002, 106:1760-1763.

17. Achilli A, Sassara M, Ficili S, et al.: Long-term effectiveness of cardiac resynchronization therapy in patients with refractory heart failure and "narrow" QRS. J Am Coll Cardiol 2003, 42:2117-2124.

18. Mollema SA, Bleeker GB, Van der Wall EE, et al.: Usefulness of QRS duration to predict response to cardiac resynchronization therapy in patients with end-stage heart failure. Am J Cardiol 2007, 100:1665-1670.

19. Bax JJ, Marwick TH, Molhoek SG, et al.: Left ventricular dyssynchrony predicts benefit of cardiac resynchronization therapy in patients with end-stage heart failure before pacemaker implantation. Am J Cardiol 2003, 92:1238-1240.

20. Yu CM, Fung WH, Lin H, et al.: Predictors of left ventricular reverse remodeling after cardiac resynchronization therapy for heart failure secondary to idiopathic dilated or ischemic cardiomyopathy. Am J Cardiol 2003, 91:684-688.

21. Chung ES, Leon AR, Tavazzi L, et al.: Results of the Predictors of Response to CRT (PROSPECT) trial. Circulation 2008, 117:2608-2616.
22. Bleeker GB, Kaandorp TA, Lamb HJ, et al.: Effect of posterolateral scar tissue on clinical and echocardiographic improvement after cardiac resynchronization therapy. Circulation 2006, 113:969-976.

23. Ypenburg C, Schalij MJ, Bleeker GB, et al.: Impact of viability and scar tissue on response to cardiac resynchronization therapy in ischaemic heart failure patients. Eur Heart J 2007, 28:33-41.

24. Adelstein EC, Saba S: Scar burden by myocardial perfusion imaging predicts echocardiographic response to cardiac resynchronization therapy in ischemic cardiomyopathy. Am Heart J 2007, 153:105-112.

25. Bilchick KC, Dimaano V, Wu KC, et al.: Magnetic resonance imaging analysis of dyssynchrony and myocardial scar predicts function class improvement following cardiac resynchronization therapy. JACC Cardiovasc Imaging 2008, 1:561-568.

26. Ypenburg C, van Bommel RJ, Delgado V, et al.: Optimal left ventricular lead position predicts reverse remodeling and survival after cardiac resynchronization therapy. J Am Coll Cardiol 2008, 52:1402-1409.

27. Kerwin WF, Botvinick EH, O'Connell JW, et al.: Ventricular contraction abnormalities in dilated cardiomyopathy: effect of biventricular pacing to correct interventricular dyssynchrony. J Am Coll Cardiol 2000, 35:1221-1227.

28. Fauchier L, Marie O, Casset-Senon D, et al.: Interventricular and intraventricular dyssynchrony in idiopathic dilated cardiomyopathy: a prognostic study with Fourier phase analysis of radionuclide angioscintigraphy. J Am Coll Cardiol 2002, 40:2022-2030.

29. Fauchier L, Marie O, Casset-Senon D, et al.: Reliability of QRS duration and morphology on surface electrocardiogram to identify ventricular dyssynchrony in patients with idiopathic dilated cardiomyopathy. Am J Cardiol 2003, 92:341-344.

30. Toussaint JF, Lavergne T, Kerrou K, et al.: Basal asynchrony and resynchronization with biventricular pacing predict long-term improvement of LV function in heart failure patients. Pacing Clin Electrophysiol 2003, 26:1815-1823.

31. Fauchier L, Eder V, Casset-Senon D, et al.: Segmental wall motion abnormalities in idiopathic dilated cardiomyopathy and their effect on prognosis. Am J Cardiol 2004, 93:1504-1509.

32. O'Connell JW, Schreck C, Moles M, et al.: A unique method by which to quantitate synchrony with equilibrium radionuclide angiography. J Nucl Cardiol 2005, 12:441-450.

33. Botvinick EH, O'Connell JW, Kadkade PP, et al.: Potential added value of three-dimensional reconstruction and display of single photon emission computed tomographic gated blood pool images. J Nucl Cardiol 1998, 5:245-255.

34. Harel F, Finnerty V, Gregoire J, et al.: Comparison of left ventricular contraction homogeneity index using SPECT gated blood pool imaging and planar phase analysis. J Nucl Cardiol 2008, 15:80-85.

35. Vilain D, Daou D, Casset-Senon D, et al.: Optimal 3-dimensional method for right and left ventricular Fourier phase analysis in electrocardiography-gated blood-pool SPECT. J Nucl Cardiol 2001, 8:371-378.

36. Muramatsu T, Matsumoto K, Nishimura S: Efficacy of the phase images in Fourier analysis using gated cardiac pool-SPECT for determining the indication for cardiac resynchronization therapy. Circ J 2005, 69:1521-1526.

37. Hoffman EJ, Huang SC, Phelps ME: Quantitation in positron emission computed tomography: 1. Effect of object size. J Comput Assist Tomogr 1979, 3:299-308.

38. Galt JR, Garcia EV, Robbins WL: Effects of myocardial wall thickness on SPECT quantification. IEEE Trans Med Imaging 1990, 9:144-150.

39. Chen J, Garcia EV, Folks RD, et al.: Onset of left ventricular mechanical contraction as determined by phase analysis of ECGgated myocardial perfusion SPECT imaging: development of a 
diagnostic tool for assessment of cardiac mechanical dyssynchrony. J Nucl Cardiol 2005, 12:687-695.

40. • Trimble MA, Velazquez EJ, Adams GL, et al.: Repeatability and reproducibility of phase analysis of gated SPECT myocardial perfusion imaging used to quantify cardiac dyssynchrony. Nucl Med Commun 2008, 29:374-381. This paper demonstrated the repeatability and reproducibility of phase analysis of gated myocardial perfusion SPECT.

41. • Chen J, Faber TL, Cooke CD, Garcia EV: Temporal resolution of multiharmonic phase analysis of ECG-gated myocardial perfusion SPECT studies. J Nucl Cardiol 2008, 15:383-391. This paper demonstrated the temporal resolution of the phase analysis of gated myocardial perfusion SPECT. It justified that the phase analysis technique has sufficient temporal resolution for measuring $L V$ dyssynchrony.

42. Trimble MA, Borges-Neto S, Smallheiser S, et al.: Evaluation of left ventricular mechanical dyssynchrony as determined by phase analysis of ECG-gated SPECT myocardial perfusion imaging in patients with left ventricular dysfunction and conduction disturbances. J Nucl Cardiol 2007, 14:298-307.

43. Trimble MA, Borges-Neto S, Honeycutt EF, et al.: Evaluation of mechanical dyssynchrony and myocardial perfusion using phase analysis of gated SPECT imaging in patients with left ventricular dysfunction. J Nucl Cardiol 2008, 15:663-670.

44. Bleeker GB, Schalij MJ, Molhoek SG, et al.: Relationship between QRS duration and left ventricular dyssynchrony in patients with end-stage heart failure. J Cardiovasc Electrophysiol 2004, 15:544-549.

45. • Henneman MM, Chen J, Ypenburg C, et al.: Phase analysis of gated myocardial perfusion single-photon emission computed tomography compared with tissue Doppler imaging for the assessment of left ventricular dyssynchrony. J Am Coll Cardiol 2007, 49:1708-1714. This paper validated gated myocardial perfusion SPECT with phase analysis against TDI and showed high correlation between $L V$ dyssynchrony measured by the two techniques.

46. Marsan NA, Henneman MM, Chen J, et al.: Left ventricular dyssynchrony assessed by two three-dimensional imaging modalities: phase analysis of gated myocardial perfusion SPECT and triplane tissue Doppler imaging. Eur J Nucl Med Mol Imaging 2008, 35:166-173.

47. Marsan NA, Henneman MM, Chen J, et al.: Real-time 3dimensional echocardiography as a novel approach to quantify left ventricular dyssynchrony: a comparison study with phase analysis of gated myocardial perfusion single photon emission computed tomography. J Am Soc Echocardiogr 2008, 21:801-807.

48. •- Henneman MM, Chen J, Dibbets-Schneider P, et al.: Can LV dyssynchrony as assessed with phase analysis on gated myocardial perfusion SPECT predicts response to CRT? J Nucl Med 2007, 48:1104-1111. This paper evaluated the sensitivity and specificity of gated myocardial perfusion SPECT with phase analysis in predicting CRT response.

49. Van Kriekinge SD, Nishina H, Ohba M, et al.: Automatic global and regional phase analysis from gated myocardial perfusion SPECT imaging: application to the characterization of ventricular contraction in patients with left bundle branch block. J Nucl Med 2008, 49:1790-1797.

50. Boogers MM, Van Kriekinge SD, Henneman MM, et al.: Quantitative gated SPECT-derived phase analysis on gated myocardial perfusion SPECT detects left ventricular dyssynchrony and predicts response to cardiac resynchronization therapy. J Nucl Med 2009, 50:718-725. 